\title{
A mechanism-based group-psychotherapy approach to aggressive behaviour in borderline personality disorder: findings from a cluster-randomised controlled trial
}

S. C. Herpertz*, B. Matzke*, K. Hillmann, C. Neukel, F. Mancke, B. Jaentsch, U. Schwenger, H. Honecker, R. Bullenkamp, S. Steinmann, M. Krauch, S. Bauer, C. Borzikowsky, K. Bertsch and A. Dempfle

\section{Background}

Aggressive behaviour is a prevalent and harmful phenomenon in patients with borderline personality disorder (BPD). However, no short-term, low-cost programme exists that specifically focuses on aggression.

\section{Aims \\ Attuning therapy modules to pathogenetic mechanisms that underlie reactive aggression in BPD, we composed a 6 week mechanism-based anti-aggression psychotherapy (MAAP) approach for the group setting, which we tested against a non-specific supportive psychotherapy (NSSP).}

\section{Method}

A cluster-randomised two-arm parallel-group phase II trial of $N=$ 59 patients with BPD and overt aggressive behaviour was performed (German Registry for Clinical Trials, DRKS00009445). The primary outcome was the externally directed overt aggression score of the Modified Overt Aggression Scale (M-OAS) posttreatment (adjusted for pre-treatment overt aggression). Secondary outcomes were M-OAS irritability, M-OAS response rate and ecological momentary assessment of anger posttreatment and at 6 month follow-up, as well as M-OAS overt aggression score at follow-up.

\section{Results}

Although no significant difference in M-OAS overt aggression between treatments was found post-treatment (adjusted difference in mean $3.49(95 \% \mathrm{Cl}-5.32$ to $12.31, P=0.22)$, the MAAP group showed a clinically relevant decrease in aggressive behaviour of $65 \%$ on average (versus $33 \%$ in the NSSP group), with particularly strong improvement among those with the highest baseline aggression. Most notably, significant differences in reduction in overt aggression between MAAP and NSSP were found at follow-up.

\section{Conclusions}

Patients with BPD and aggressive behaviour benefited from a short group psychotherapy, with improvements particularly visible at 6 month follow-up. Further studies are required to show whether these effects are specific to MAAP.

\section{Keywords}

Group psychotherapy; dialectic behaviour therapy; mentalisation-based therapy; anger regulation; threat hypersensitivity; emotional contagion.

\section{Copyright and usage}

(c) The Author(s), 2020. Published by Cambridge University Press on behalf of the Royal College of Psychiatrists. This is an Open Access article, distributed under the terms of the Creative Commons Attribution-NonCommercial-ShareAlike licence (http://creativecommons.org/licenses/by-nc-sa/4.0/), which permits non-commercial re-use, distribution, and reproduction in any medium, provided the same creative Commons licence is included and the original work is properly cited. The written permission of Cambridge University Press must be obtained for commercial re-use.

\section{Introduction}

Aggression is a highly prevalent and harmful phenomenon in female and male patients with borderline personality disorder (BPD). ${ }^{1}$ However, to date, no treatment programmes have been developed that specifically focus on reducing aggression in BPD.

In most randomised controlled trials (RCTs) in BPD to date, self-harm, (para)suicidal behaviour and general symptom severity have been the outcome measures. In these studies, dialectical behaviour therapy (DBT) and psychodynamic approaches were found to be more effective than control interventions. ${ }^{2,3}$ Aggression was the focus of democratic therapeutic community treatment, a group therapy delivered for between 5 and $15 \mathrm{~h}$ per week over a maximum of 1.5 years, compared with treatment as usual (TAU) in BPD. It significantly reduced aggression assessed with total Modified Overt Aggression Scale (M-OAS) ${ }^{4}$ score 2 years but not 1 year after baseline. ${ }^{5}$ Pre- to post-treatment reduction of aggression, again measured with the M-OAS, followed a 1 year

* Joint first authors
DBT treatment of patients with BPD and antisocial behaviour in a within-group design without comparator treatment. ${ }^{6}$ Furthermore, anger decreased after DBT skills training as a standalone treatment but hostility did not. ${ }^{7,8}$ Mentalisation-based therapy (MBT) reduced anger and hostility in out-patients with BPD and comorbid antisocial personality disorder when offered as weekly combined individual and group psychotherapy over 18 months compared with structured clinical management. ${ }^{9}$ Significant effects were found after 18 months but not after 6 or 12 months of treatment. In summary, DBT and MBT programmes have shown some promising effects in reducing aggression and anger in BPD; however, targeted, cost-effective programmes are urgently needed. Psychotherapy for BPD, such as skills training, has often been conducted in a group format, and recently published Cochrane meta-analyses ${ }^{3}$ provide evidence for the efficiency of group psychotherapy in BPD. Small-group psychotherapy in aggressive adolescents has been shown to facilitate options for practice of social and emotional skills and to reinforce adaptive behaviours; ${ }^{10}$ however, the differential effects of individual and group psychotherapy have not yet been systematically compared in BPD.

Aggression in BPD is usually triggered by real or perceived social threat, frustration or social provocation. ${ }^{11}$ Based on 
behavioural and neurobiological data from our own and other research groups, we recently proposed a model according to which reactive aggression in BPD arises from several mechanisms: ${ }^{12}$ (a) social threat hypersensitivity, that is, hypervigilance to social threat cues, biased negative perception of others, and a failure to recognise social safety signals; ${ }^{13}$ (b) approach rather than avoidance of social threat cues; ${ }^{14}$ (c) maladaptive anger regulation, ${ }^{15}$ with emotion dysregulation and anger sequentially mediating the relationship between BPD and aggression; ${ }^{16}$ (d) low capacity to adequately mentalise the intentions, cognitions and emotions of others; ${ }^{17}$ and (e) excessive emotional imitation and contagion. ${ }^{18}$ Notably, these mechanisms interact with one another, e.g. perceived rejection is strongly interrelated with anger, ${ }^{19}$ and low mentalising capacity is associated with social threat hypersensitivity ${ }^{20}$ and poor emotion regulation capacity. ${ }^{21}$

Starting from the theory that therapy development should be attuned to the pathogenetic mechanisms which underlie a specific psychopathology, ${ }^{22}$ we composed an aggression-specific psychotherapeutic intervention programme called mechanism-based anti-aggression psychotherapy (MAAP) for the group setting that specifically targets these mechanisms. This programme includes techniques from evidence-based treatment programmes for BPD that focus on the mechanisms summarised above, namely, the well-established skills training from $\mathrm{DBT}^{23}$ and interventions from MBT. ${ }^{24}$ Targeted interventions using DBT and MBT were complemented by specific app-based attention training techniques $^{25}$ in order to reduce attentional bias to threat cues and instead foster vigilance with respect to safety signals. The interventions have been compiled in such a way as to target the identified mechanisms, while being focused and allowing a high frequency of repetition within a short, low-cost intervention.

In this RCT, we tested the hypothesis that MAAP is superior to non-specific supportive psychotherapy (NSSP) in terms of reducing aggressive behaviours and anger in BPD at the end of therapy as well as at 6 month follow-up.

\section{Method}

\section{Trial design and participants}

The efficacy of MAAP was compared to that of NSSP in a clusterrandomised two-arm parallel-group phase II trial (allocation ratio of $1: 1$, with psychotherapy groups as clusters), performed at the Psychiatric University Hospital, Heidelberg, Germany. The trial was approved by the Ethics Committee of the Medical Faculty, Heidelberg. Before initiation, the study was registered in a public trial archive (German Registry for Clinical Trials, DRKS00009445). Written informed consent was obtained from all patients who were included between March 2016 and January 2019 (for details of recruitment and inclusion and exclusion criteria, see the supplementary material available at https://doi.org/bjo.2020.131).

Out-patients (18-55 years of age) with BPD were recruited by referrals from psychiatrists, psychotherapists and psychiatric hospitals, as well as via the internet (study website, postings and advertisements on Facebook, Google and eBay classifieds), newspapers, radio reports, flyers, and registers of previous study participants. Participants had to meet at least four BPD criteria according to the International Personality Disorder Examination (IPDE) (therefore also including subthreshold BPD), overt aggression (subscales $1-3 \geq 6$ ) and irritability (subscales $5-6 \geq 6$ ) over 2 weeks according to the M-OAS. During the course of the study, the maximum age for inclusion was raised from 50 to 55 years, facilitating the recruitment of the sample size needed to reach the defaults of the power analysis.

\section{Assessments}

Two research diagnosticians, who were blind to the treatment delivered, conducted assessments at inclusion, at pre- and posttreatment time points, and 6 months after therapy had ended. They had received training in the M-OAS, ${ }^{26}$ which is an interview-based measure to assess frequency and severity of overt aggressive behaviour occurring in a time frame of 2 weeks and is sensitive to change ${ }^{27}$ (see the supplementary material). It consists of three subscales: an aggression subscale (comprising verbal attacks (item 1 ), assaults against objects (item 2) and other people (item 3), and assaults against self (item 4)); an irritability subscale (subjective (item 5) and objective (item 6)); and a suicidal tendencies subscale (items 7 to $7 \mathrm{~b}$ ). All M-OAS item scores have a minimum value of zero; however, items 1-4 have no upper limit on their maximum value. As we were interested in externally directed overt aggression only, and not auto-aggressive, self-harming behaviours (as in many previous studies of $\mathrm{BPD}^{2}$ ), the primary outcome was based on items 1-3 of the M-OAS in this study. Detailed explanation of the primary outcome in the German Registry for Clinical Trials (DRKS00009445) is not provided (clarifications of the protocol can be accessed in the supplementary material). Ecological momentary assessments (EMA) took place over the course of 2 weeks, three times a day (in the morning, at midday and in the evening at random times). The EMA included a rating of current negative emotional arousal on a visual analogue scale between 0 and 100, the STAXI-State Anger Scale, and the choice of dominant affect among eight alternatives (joy, anxiety, sadness, shame, guilt, disgust, contempt and anger).

In addition to measuring change in symptoms, behavioural laboratory and neuroimaging assessments were conducted before and after treatment in order to investigate treatment-related changes in biobehavioural mechanisms (not reported here).

At inclusion, the IPDE ${ }^{28}$ based on the DSM-IV was performed to assess the inclusion and exclusion criteria for BPD. The Structured Clinical Interview for Mental Disorders (SCID-I) was used to assess comorbid current and lifetime psychiatric disorders, the Global Assessment of Functioning Scale (GAF) was used to assess the social, occupational and psychological functioning of an individual, the Zanarini Rating Scale for Borderline Personality Disorder $(\mathrm{ZAN})^{29}$ was used to assess borderline symptom severity over the past week, and Raven's Progressive Matrices ${ }^{30}$ were administered to estimate intelligence. Finally, a self-report measure of aggressive behaviours, the Life History of Aggression Scale ${ }^{31}$ was used.

\section{Outcome measures}

The M-OAS externally directed overt aggression score (items 1-3) post-treatment was selected as the primary outcome measure. Secondary outcomes were M-OAS irritability (items 5 and 6), MOAS response rate (reduction of M-OAS overt aggression score (items $1-3$ ) by at least $50 \%$ from baseline) and EMA (STAXIState Anger Scale, emotional arousal and the percentage of assessments where anger was the dominant affect) post-treatment and at 6 month follow-up, as well as M-OAS overt aggression score at follow-up. All outcomes were measured at the level of the individual patient, not at the therapy group level (cluster level). To minimise missing data (especially on the primary outcome), patients were asked to participate in outcome assessments even if they had discontinued therapy.

\section{Interventions}

Both types of intervention were conducted as group therapy, starting with one individual $1 \mathrm{~h}$ session followed by 6 weeks of group therapy with two $1.5 \mathrm{~h}$ sessions per week (a total of $18 \mathrm{~h}$ ). The 
study protocol required that there were no changes in psychotropic medication during treatment and that participants attended at least eight of the 12 planned treatment sessions. One therapy group consisted of three to six patients (mean: 4.08 patients) and two trained therapists. We ran 12 therapy groups in total (six MAAP groups and six NSSP groups). One further MAAP therapy group was cancelled owing to drop-out of all but one patient before the group started (see the supplementary material for details of training and supervision).

\section{Mechanism-based anti-aggression therapy}

MAAP is a highly structured manualised programme (for an overview of the intervention, together with its aims and its targets, see Fig. 1 in the supplementary material available at https:/doi.org/ bjo.2020.131). The aim of the first individual session was to motivate change, to explain the model of reactive aggression and treatment principles, and to work out an individual crisis plan. Two app-based attentional exercises developed for this study and intended to be performed as homework on 2 days per week between sessions, were explained to patients. One of them, a visual search exercise, aimed to reduce hypervigilance to social threat through focused attention toward social cues indicating safety. Participants were instructed to find the only friendly looking face in a gathering of frowning or at least neutral faces. ${ }^{32}$ The other exercise instructed participants to find hidden smiling faces and to differentiate them from hidden threatening faces. Both exercises were ordered by increasing degree of difficulty, with nine trials performed at one time.

In group session 1, the exercises involved identifying individual internal and external triggers for aggressive behaviours and analysing the negative effects of these behaviours. Between-session exercises dealt with identifying triggers in everyday life. In session 2 , cognitive, emotional, physical and behavioural correlates of anger were worked out among group members, and contextual factors were identified that enhance the risk of aggression. DBT emotion regulation techniques were introduced to enable the individual to monitor their own emotions with regard to intensity, bodily reactions, consecutive cognitions and behaviours and to increase selfawareness of triggers that initiate anger. The homework was to apply the learnt techniques to the most alarming event of the day. Session 3 focused on cognitive schemata and automatic thoughts, and collected learning experiences of the group members that reinforced feelings of anger and aggressive behaviours. Session 4 dealt with various DBT anger regulation skills, integrating physical and imagination exercises to dampen anger and increase positive feelings. Opposite facial actions (e.g. slight smile, raised eyebrows) were introduced to create sensory feedback ${ }^{33}$ and to dampen emotion contagion. Session 5 provided guidance in discrimination exercises that help to differentiate actual situational triggers from the activation of cognitive schemata learned in the past. Session 6 dealt with mindfulness-based perception and introduced the construct of mentalising according to MBT. The group was instructed to reflect on how behaviour and mental attributions to oneself and others interrelate. Session 7 aimed to replace automatic, reflexive schema-driven mentalising with controlled reflective mentalising. The therapists inspired exchange among patients about inner monologues evoked by images of social scenes and about how to understand another patient's affect within group situations. In sessions 8 to 11 , all members presented with scripts of real-life situations and associated inner monologues. In session 12, group members summarised the take-home messages and updated their crisis management plans.

\section{Non-specific supportive psychotherapy}

NSSP (J. Markowitz, M. Sacks, A. Frances, personal communication, 2002), as the manualised comparator treatment, was parallelised in dosage (one individual session and 12 group sessions). It has been compared with DBT in several studies in the group setting ${ }^{8}$ and focuses on the non-specific or 'common' factors assumed to be important ingredients across psychotherapies, including psychoeducational elements and a therapist's roles of reflective listening, empathy, facilitating affect, therapeutic optimism and acknowledgment of patients' resources. Therapists encourage patients to find answers within themselves instead of offering specific skills, and refrain from using specific cognitive, behavioural or psychodynamic interventions.

\section{Sample size}

In an a priori power analysis based on the effect size of a DBT skills group, ${ }^{34}$ a sample size of $N=25$ per treatment arm was found to be sufficient to detect medium-sized interaction effects (time point $x$ treatment) of $\Omega^{2}=0.10$ with power of $1-\beta=0.80$ and two-sided significance level of 0.05 . This sample size calculation was confirmed by a meta-analysis of group psychotherapy in BPD published in 2019, indicating large effects (Cohen's $\mathrm{d}=0.8$ ), ${ }^{35,36}$ for which this sample size would have $80 \%$ power. As patients with BPD are a difficult-to-treat population, with only moderate adherence and relevant loss to follow-up in previously published trials, ${ }^{5,37-39}$ we expected missing data on the primary outcome in $20 \%$ of randomised patients and thus increased the total sample size to 59 patients.

\section{Randomisation}

The unit of randomisation was the therapy group, not the individual patient. An independent research assistant (R.B.), who was not involved in either screening and diagnosis or therapy, performed the cluster randomisation in a $1: 1$ ratio by throwing a coin. Sometimes, only therapists for one treatment arm were available (e.g. during vacation times), the available tandem of psychotherapists was then deterministically assigned for the next therapy group. In all cases, diagnosticians were informed about the starting date of the next group without being informed which treatment arm was the next to start (concealment of allocation). Patients were informed about two different treatments at the time of study enrolment, without indicating that there was an experimental and a comparator treatment.

\section{Statistical methods}

The superiority of the primary outcome measure in the MAAP arm over the NSSP arm was tested by a mixed linear model of posttreatment overt aggression score as the dependent variable, pretreatment overt aggression score as a covariate, treatment arm as a fixed effect and therapy group as a random effect. The primary analysis was based on slightly modified intention-to-treat (mITT) principles: we did not include patients who withdrew consent, had not participated in any of the randomised treatments (dropout between inclusion and treatment start), or had neither posttreatment nor follow-up data available because they did not take part in assessments. An analysis of patients treated per protocol included only those with no change in psychotropic medication and attending at least eight out of 12 therapy sessions (for details, see the supplementary material)

\section{Results}

\section{Flow of participants over the trial}

The flow of participants is illustrated in Fig. 1. Of a total of 336 patients assessed for eligibility, 92 were positively screened and 59 fulfilled all 


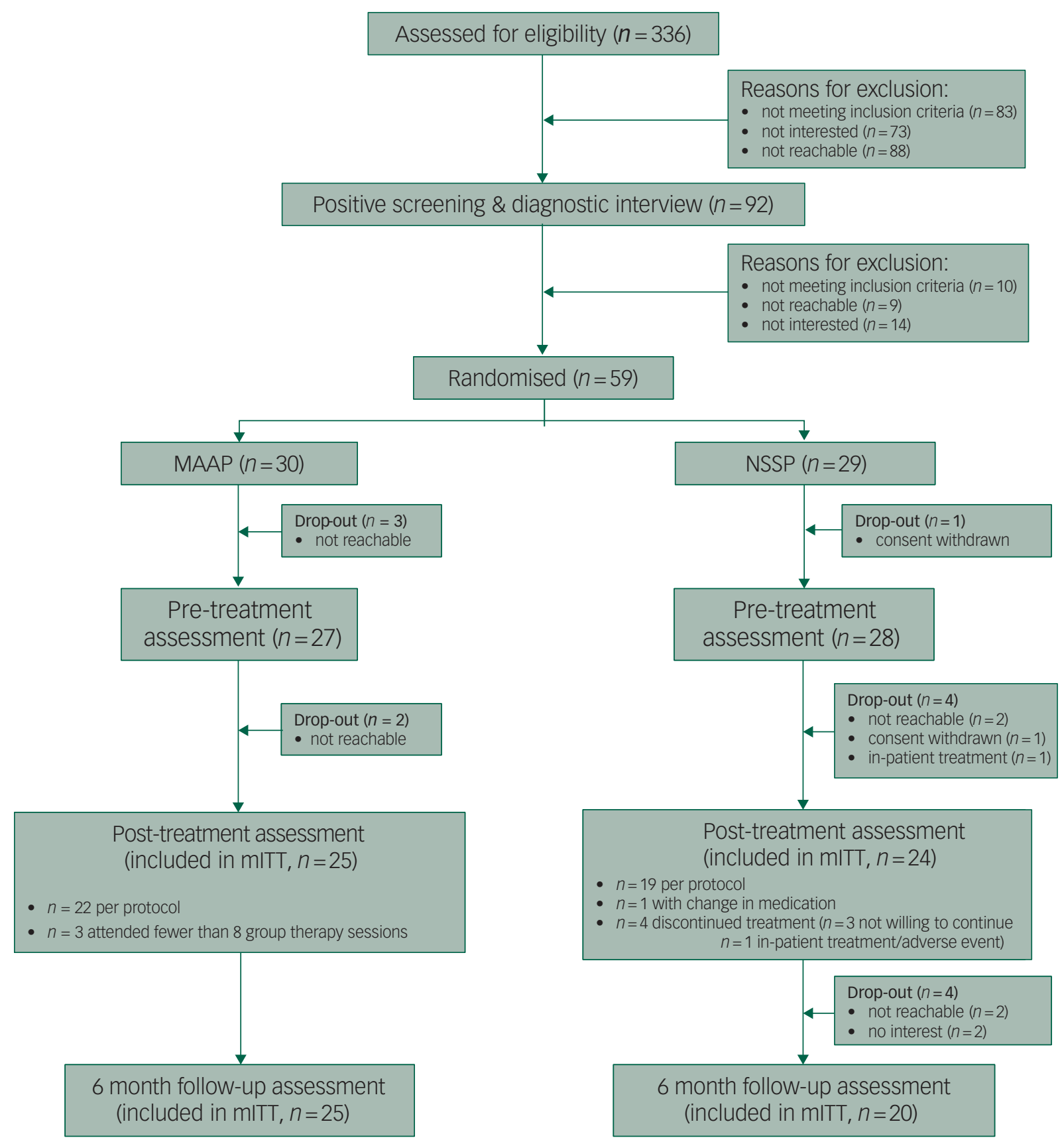

Fig. 1 Trial design and flow of patients through the inclusion, pre-treatment, post-treatment and follow-up measurement points.

inclusion criteria and consented to participate. In order to facilitate recruitment, we removed current cannabis misuse as an exclusion criterion and extended the age range from 18 to 55 years over the course of the study. Of those patients fulfilling the inclusion criteria, $30(66.7 \%$ female) were allocated to six MAAP therapy groups and 29 (62.1\% female) to the comparator NSSP groups (also six therapy groups) between January 2016 and January 2019. Four patients did not start the intervention (and were not included in the mITT set), and ten patients discontinued treatment (two from the MAAP group and eight from the NSSP group), six of whom did not undergo the post-treatment assessment (also not included in the mITT set). Thus, 25 patients from the MAAP group ( $83.3 \%$ of those initially randomised) and 24 patients from the NSSP group ( $82.8 \%$ of those initially randomised) were included in the mITT analyses. Three patients from the MAAP group and one patient from the NSSP group showed relevant protocol violations, namely, attending fewer than eight therapy sessions or having a change of medication, which resulted in 22 patients from the MAAP and 19 patients from the NSSP group being available for per-protocol analyses. All 25 patients from the MAAP arm could be reached for the 6 month follow-up assessment, while four patients from the NSSP group were lost to follow-up.

Two patients in the NSSP arm experienced emergency hospital admissions during group therapy (serious adverse events, both crisis interventions related to $\mathrm{BPD}$ ), whereas no serious adverse event occurred in the MAAP treatment arm.

Clinical characteristics at the time of inclusion are provided in Table 1. The sample was comparable with other clinical samples ${ }^{6,40,41}$ in terms of symptom severity, psychiatric comorbidities, medication and age; thus, it represented a typical treatmentseeking BPD out-patient population of moderate severity, given some recently published trials showing higher ZAN and GAF scores. ${ }^{39,42}$ Owing to the treatment focus on aggression, the 


\begin{tabular}{|c|c|c|c|c|}
\hline & \multicolumn{2}{|c|}{ MAAP $(n=30)$} & \multicolumn{2}{|c|}{$\operatorname{NSSP}(n=29)$} \\
\hline & \multicolumn{2}{|c|}{ mean (s.d.) or $n(\%)$} & \multicolumn{2}{|c|}{ mean (s.d.) or $n(\%)$} \\
\hline Age (range: $18-53$ years in MAAP group, $21-55$ years in NSSP group) & & $29.77(9.50)$ & & $33.24(8.76)$ \\
\hline Gender (female) & & $20(66.7)$ & & $18(62.1)$ \\
\hline Current psychotropic medication & & $9(31.0)^{a}$ & & $13(44.8)$ \\
\hline Antidepressants & & $7(24.1)^{\mathrm{a}}$ & & $11(37.9)$ \\
\hline Neuroleptics & & $3(10.3)^{a}$ & & $4(13.8)$ \\
\hline Other & & $2(6.9)^{a}$ & & $4(13.8)$ \\
\hline Comorbidities & current & lifetime & current & lifetime \\
\hline Major depression & $7(24.1)^{\mathrm{a}}$ & $20(69.0)^{\mathrm{a}}$ & $9(32.1)^{\mathrm{a}}$ & $22(78.6)^{a}$ \\
\hline Dysthymia & $5(16.7)^{a}$ & - & $1(3.8)^{a}$ & - \\
\hline Alcohol addiction/misuse & $0(0.0)^{a}$ & $6(20.7)$ & $0(0.0)^{\mathrm{a}}$ & $6(22.2)^{a}$ \\
\hline Anxiety disorders & $12(42.9)^{a}$ & $11(39.3)^{a}$ & $11(39.3)^{\mathrm{a}}$ & $14(50.0)^{a}$ \\
\hline Obsessive-compulsive disorder & $2(7.1)^{a}$ & $2(7.1)^{a}$ & $1(3.6)^{a}$ & $2(7.1)^{a}$ \\
\hline Post-traumatic stress disorder & $10(35.7)^{a}$ & $10(35.7)^{a}$ & $8(28.6)^{a}$ & $7(25.0)^{a}$ \\
\hline Somatisation disorder & $1(3.4)^{\mathrm{a}}$ & - & $0(0.0)^{a}$ & - \\
\hline Eating disorder & $1(3.4)^{\mathrm{a}}$ & $6(20.7)^{a}$ & $2(7.1)^{\mathrm{a}}$ & $6(21.4)^{a}$ \\
\hline Antisocial personality disorder & $5(16.7)$ & $9(30.0)^{\mathrm{a}}$ & $1(3.7)^{\mathrm{a}}$ & $5(17.9)^{a}$ \\
\hline Avoidant personality disorder & $8(26.7)$ & $7(23.3)$ & $10(35.7)^{\mathrm{a}}$ & $8(28.6)^{a}$ \\
\hline Mean number of comorbid disorders & $2.21(1.52)$ & $3.43(1.95)$ & $1.89(1.55)$ & $3.18(1.72)$ \\
\hline Number of BPD DSM-5 criteria (range: $4-9)^{b}$ & & $6.63(1.22)$ & & $5.46(1.17)$ \\
\hline ZAN rating scale for BPD (global sum) & & $13.47(4.66)$ & & $12.08(3.10)$ \\
\hline ZAN subscale: affective disorder & & $5.60(2.21)$ & & $5.00(1.17)$ \\
\hline ZAN-subscale: cognitive disorder & & $2.73(1.76)$ & & $1.92(1.79)$ \\
\hline ZAN-subscale: impulsiveness & & $2.48(1.79)$ & & $2.04(1.31)$ \\
\hline ZAN-subscale: relationship problems & & $2.70(1.44)$ & & $3.00(1.23)$ \\
\hline M-OAS at inclusion & mea & ange) & mea & ange) \\
\hline Total $^{c}$ & 63.57 & 18-191) & 42.31 & 14-95) \\
\hline Overt aggression (items 1-3) & 40.10 & 7-142) & 28.3 & $6-80)$ \\
\hline Self-injury (item 4) & & $14.70(25.46)$ & & $5.79(10.17)$ \\
\hline Irritability (items 5-6) & & $6.97(0.93)$ & & $6.90(0.82)$ \\
\hline Suicidal tendency (items 7; 7b) & & $1.80(1.30)$ & & $1.28(1.10)$ \\
\hline \multicolumn{5}{|l|}{ Life History of Aggression Scale } \\
\hline Total score & & $29.88(9.91)$ & & $27.85(9.26)$ \\
\hline Aggression & & $18.92(4.92)$ & & $18.56(4.48)$ \\
\hline Antisocial & & $5.83(4.50)$ & & $3.96(4.47)$ \\
\hline Self-aggression & & $5.13(2.82)$ & & $5.33(3.16)$ \\
\hline Intelligence quotient (IQ Raven) & & $99.24(16.13)$ & & 99.58 (14.37) \\
\hline Global Assessment of Functioning (GAF) & & $51.48(7.80)$ & & $52.30(13.25)$ \\
\hline
\end{tabular}

percentage of male participants was higher than in most clinical trials in BPD. Despite random allocation to the treatment arm, the two therapy groups differed in severity of psychopathology at inclusion: patients assigned to the MAAP group presented with higher symptom severity on almost all of the BPD measures, in particular, significantly higher M-OAS total scores and higher severity of BPD psychopathology; the maximum value of the M-OAS overt aggression scale at inclusion was 80 in the NSSP arm (maximum total M-OAS 95) and 142 in the MAAP arm (maximum total M-OAS 191). At the time point immediately before treatment start (pre-treatment), seven of the 25 MAAP patients available for mITT analysis had an M-OAS overt aggression value between 61 and 132, which was larger than the maximum value of 60 among all 24 NSSP patients (Fig. 2(a)); at this time point, the mean value in the MAAP group was 38.6, while that in the NSSP group was 19.2. Owing to non-specific natural shifts in severity of symptoms (and a possible regression-to-the-mean effect), we found a mean difference in overt aggression score between inclusion and pretreatment assessments of 6.67 (median 11) in the overall group (a $19 \%$ decline).

\section{Outcomes}

The outcomes are summarised in Table 2. In the mITT analyses, we found no significant treatment effect on the primary outcome, the
M-OAS overt aggression score comprising verbal and physical aggression against objects and others, at the post-treatment time point $(P=0.22$ in the mixed model analysis of log-transformed values, adjusted for pre-treatment score and with a random intercept term for the therapy group). The adjusted difference (on the original scale) between treatment arms in mean M-OAS overt aggression was 3.49 (95\% CI -5.32 to $12.31, P=0.22, \mathrm{~F}=1.72$ ). Boxplots of overt aggression of each patient grouped according to time point and treatment arm are presented in Fig. 2(a). The difference between pre- and post-treatment M-OAS overt aggression scores in the MAAP group was 25.21 (median 13), which corresponds to a $65 \%$ decline. In the NSSP group, this unadjusted mean decrease was only 6.33 (median 4.5), representing a 33\% decline.

There was a significant effect of pre-treatment overt aggression on post-treatment aggressive behaviour $(P<0.001)$, with those patients with higher pre-treatment scores experiencing a larger reduction, while those with low baseline aggression showed little change (Fig. 2(b)). Correlation of overt aggression scores within therapy groups was small post-treatment and at follow-up (IntraClass-Correlation 0.075 and 0.007 , respectively). The apparent difference in (unadjusted) improvement was particularly driven by the most severely affected patients (high pre-treatment overt aggression scores), who were overrepresented in the MAAP arm. Regarding individuals, only one patient in the MAAP group and nine patients 

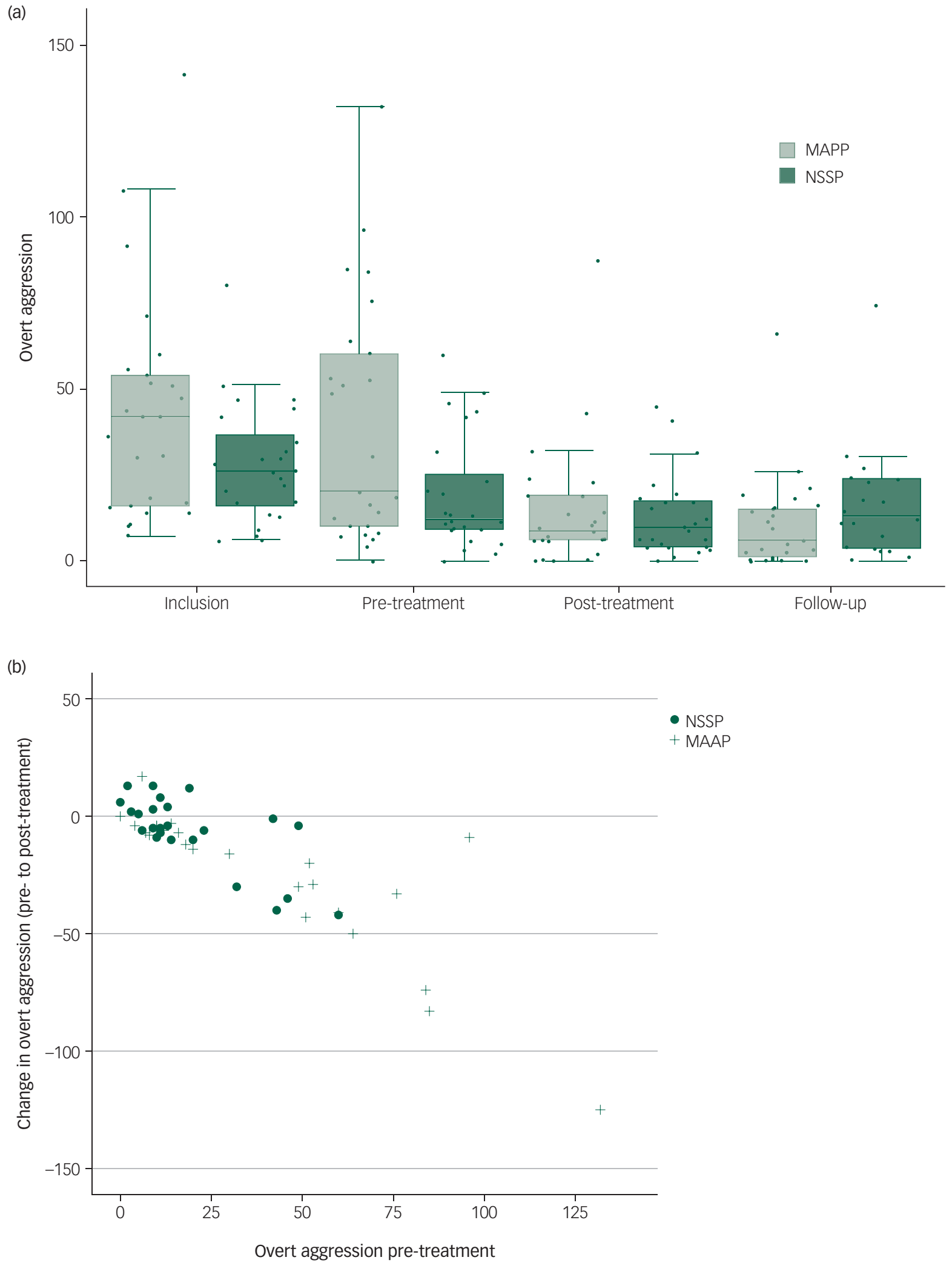

Fig. 2 (a) Boxplots of the primary outcome of overt aggression (items 1-3 of the M-OAS) for each patient grouped according to time point and treatment arm. (b) Differences in overt aggression (items 1-3 of the M-OAS) between pre- and post-treatment time points for each patient according to treatment arm and pre-treatment M-OAS value.

in the NSSP group exhibited an increase in aggressive behaviours at the end of treatment (Fig. 2(b)).

We did not find superiority of MAAP compared with NSSP treatment on any secondary outcome post-treatment; the EMA measurement of negative emotional arousal tended to decline more in the MAAP than in the NSSP therapy group $(P=0.056)$.

At 6 month follow-up, between-group differences became evident. Patients treated in the MAAP group showed less overt 


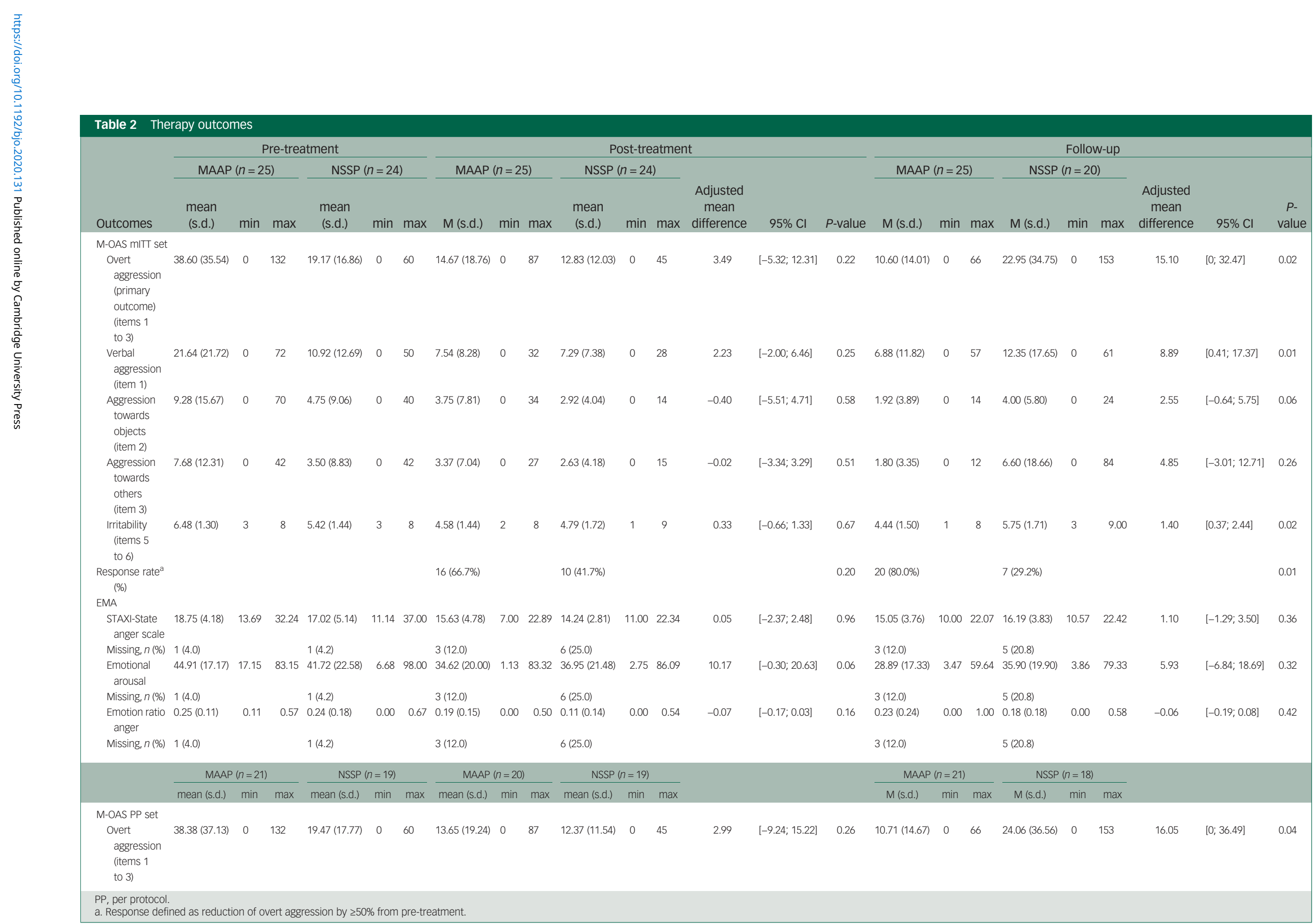


aggression (with a further decrease to a mean score of 10.6) compared with participants in the NSSP group (with an increase to 22.95, adjusted difference in means between arms 15.10 ; $95 \%$ CI 0 to $32.47, P=0.018, \mathrm{~F}=8.41$ ). $\mathrm{M}$-OAS verbal aggression and irritability were more improved in the MAAP group than in the NSSP group ( $P=0.01$ and $P=0.02$, respectively) and a higher response rate was found $(P=0.01)$. Whereas only $29.2 \%$ of individuals in the comparator group fulfilled the response criterion, $80 \%$ of the patients who had been treated with MAAP were found to be responders at follow-up assessment. No differences in EMA measurements between therapy groups were found at follow-up.

Per-protocol analyses led to similar findings (Table 2).

\section{Discussion}

This was the first RCT in patients with BPD to directly target the clinically important and disturbing problem of aggressive behaviours in this population. Based on biobehavioural mechanisms which underlie reactive aggression in $\mathrm{BPD},{ }^{12}$ we composed a short-term, low-cost aggression-specific psychotherapeutic intervention programme for the group setting, MAAP, which we tested against an active but non-specific control treatment of supportive psychotherapy (NSSP). MAAP was significantly superior to NSSP at 6 month follow-up according to our primary outcome measure, overt aggression, but not directly at the post-treatment time point. The results have to be interpreted with caution owing to relevant group differences in overt aggression at the pretreatment time point.

Although no significant difference between therapy groups was found at the end of treatment based on the frequency and severity of aggression according to the M-OAS, patients included in the MAAP therapy group showed a clinically highly relevant decrease in overt aggressive behaviour of $65 \%$ on average, with a particularly strong improvement among those with the highest baseline aggression levels. This effect on overt aggression (as measured by the M-OAS) is equivalent to or even higher than those reported by RCTs of psychopharmacological agents, such as the aggression-reducing effect of divalproex in cluster B personality disorders (a $46.8 \%$ reduction) ${ }^{43}$ or of clozapine in adolescents with conduct disorder (a 58.4\% reduction). ${ }^{44}$ To our knowledge, there have been only two psychotherapy RCTs with overt aggression as the therapy target. One, which offered a high-dose group therapy, namely democratic therapeutic community treatment, for up to 18 months to individuals with personality disorders (mostly BPD), reported a significantly higher decrease in M-OAS score 24 months after randomisation compared with TAU, with a $60 \%$ decrease from pre-therapy; however, it failed to show significant improvement after 12 months. ${ }^{5}$ Another RCT found the M-OAS score to decrease by $43.1 \%$ following Williams life skills training ${ }^{45}$ in young male criminal offenders in a prison setting. ${ }^{46}$ Interestingly, this cognitive-behavioural approach shares some principles with MAAP as it focuses on improving social cognitive information processing and subsumes training of skills that serve to change aggressive emotions and thoughts.

The high variability in externally directed overt aggression M-OAS scores, as well as the difference between the treatment arms prior to therapy despite randomised assignment to therapy groups, may have made significant results more difficult to find. However, in the NSSP group, changes from start to end of treatment were of comparable size with changes over the approximately 3-4 weeks between inclusion in the study and the start of group therapy (without any specific treatment), while there was a much greater decline in aggressive behaviour during MAAP treatment than during the wait. This makes it unlikely that the decrement in overt aggression reflects random natural fluctuations alone.
However, our data do not allow us to conclude that this observed decline in aggressive behaviour was specific to the MAAP treatment, since treatment effects appear to be positively correlated with the severity of aggressive behaviour, as reported by previous psychotherapy studies in BPD that demonstrated clinical severity to predict good treatment response. ${ }^{47,48}$ We cannot exclude the possibility that the small effect of the control treatment was related to the lower severity of aggression at treatment start. Nevertheless, some aspects suggest the superiority of MAAP over NSSP. (a) In addition to the greater mean score decrement in aggressive behaviour in the MAAP group compared with the NSSP group, deterioration was found in only one patient who had received MAAP, whereas nine patients showed more or less increased aggression with the active comparator treatment. (b) EMA as a secondary outcome measurement indicated a larger decrease in negative emotional arousal in the MAAP compared with the NSSP treatment arm. (c) Higher adherence and a lower discontinuation rate in the MAAP compared with the NSSP group may point to patients' higher approval of the experimental treatment. (d) Most notably, at follow-up, significant differences in reduction in overt aggression between the MAAP and NSSP groups were found (according to mITT and per-protocol analyses, adjusted for baseline imbalance), and the number of treatment responders was higher in the MAAP group. As irritability at follow-up was also significantly lower in those who had attended the MAAP group, this programme may have better improved capacity for affect regulation compared with the control treatment. The stable or slight increase in improvement at follow-up in the MAAP group, in contrast with a worsening in the NSSP group, suggests a stable treatment effect and points to the initiation of a successful learning process that brings about change in psychopathology beyond non-specific effects during treatment.

Strengths of the study design included a primary outcome measurement based on self-report of actual aggressive incidents in a structured interview, weighted according to their severity, rather than process measures such as anger or hostility. Furthermore, not only patients but also therapists who were comparable with regard to professional experience were randomly assigned to the two active treatment arms (see the supplementary material). This procedure counteracts critiques that followers of new therapies are likely to be more enthusiastic and to bond with patients particularly well compared with those offering the standard comparator therapy. ${ }^{49}$ Furthermore, low-dose psychotherapy in a group format makes implementation in mental healthcare services easier and probably harnesses options for practice of emotional and mentalising skills. ${ }^{10}$ Missing data due to loss to follow-up was moderate (about 17\%) considering the difficulty of follow-up in this patient population with $\mathrm{BPD}$, and lower than in other trials. ${ }^{5,42,50}$ The main limiting factor was the imbalance in baseline M-OAS score between the treatment arms, which may have biased the results. We assume that this imbalance occurred owing to chance in this small sample size. The large improvement in the most severely affected patients (all in the MAAP arm) might have been due to regression to the mean, but since such a large spontaneous improvement would be unexpected in this patient population, it is more likely to reflect true therapy effects of MAAP. Ceiling effects might also have contributed to our result: even though all patients showed aggressive behaviour at inclusion into the study (overt aggression score greater than or equal to 6, with only five patients scoring below 10 ), there were 14 patients with relatively moderate levels of aggressive behaviour (scores between 0 and 10) at the pre-treatment time point. Consequently, patient recruitment in a phase III multi-centre study should only include patients who exhibit an overt aggression score higher than 15 . Despite successful recruitment according to sample size calculation and well-estimated drop out $(<20 \%)$, a further limitation was the 
rather small sample size in light of the high variability in patients' outcomes. Finally, we did not assess the time period since onset of BPD or since first treatment; these are factors that may have modulated treatment prognosis.

In conclusion, patients with BPD and severe aggressive behaviour benefited from a new 6 week intensive mechanism-based psychotherapy in a group setting, with improvements particularly visible at 6 month follow-up. This is a remarkable result, as aggression is a hard-to-change symptom leading to much suffering for the patient and for society. Neuroimaging and experimental data assessed before and after treatment for participants in both therapy groups will hopefully elucidate whether MAAP worked through the mechanisms we hypothesised to bring about change. Mechanism-based treatment protocols are more likely to parallel the new dimensional approaches to classifying personality disorders, since mechanisms mediate functional impairments rather than categorical disorders.

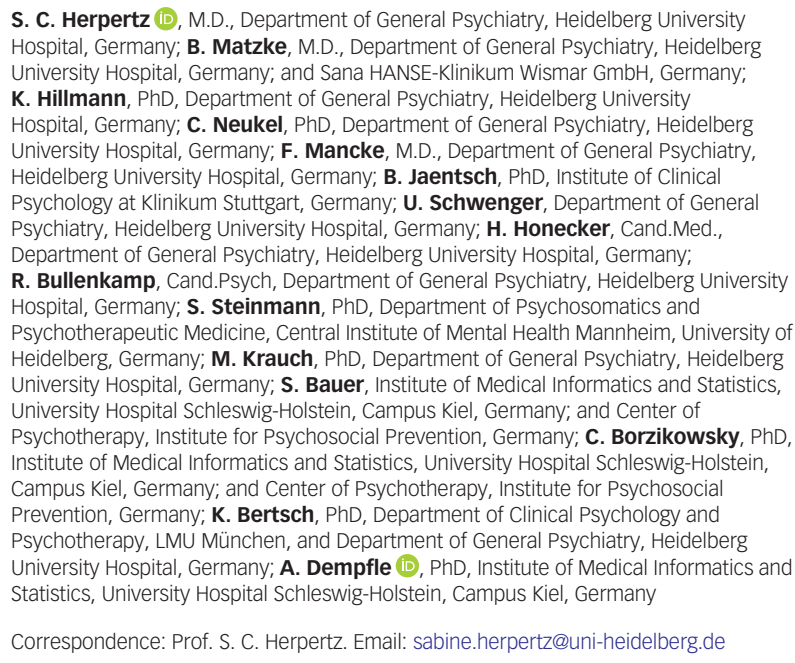

\section{Supplementary material}

Supplementary material is available online at http://doi.org/10.1192/bjo.2020.131.

\section{Data availability}

The data that support the findings of this study are available from the corresponding author, S.C.H., upon reasonable request.

\section{Author contributions}

S.C.H. together with K.B. and U.S. designed the study and provided substantial contributions to interpretation of the data S. C. drafted the work B.M was responsible for the development of the MAAP treatment as well as training and acquisition of data; he revised the manuscript. K.H., C.N. R.B., H.H., C.S., and M.K. provided substantial contributions to data acquisition, and K.H. also drafted parts of the manuscript. S.B. conceptualised and realised the EMA. A.D. and C.B. performed the data analyses, with A.D. also contributing substantially to the interpretation of the data. All authors had access to the study data and revised the work critically for important intellectual content. They gave final approval of the manuscript and agreed to be accountable for all aspects of the work

\section{Funding}

This study was part of the Clinical Research Group KFO 256 on the 'Mechanisms of Disturbed Emotion Processing in Borderline Personality Disorder' (spokesperson: Dr Schmahl, deputy spokesperson: S.C.H.) supported by the German Research Foundation. S.C.H. and K.B. report having received funding for this project (DFG; HE 2660/12-2).

\section{Declaration of interest}

ICMJE forms are in the supplementary material, available online at http://doi.org/10.1192/bjo. 2020.131.

\section{References}

1 Newhill CE, Eack SM, Mulvey EP. Violent behavior in borderline personality. J Pers Disord 2009; 23(6): 541-54.

2 Cristea IA, Gentili C, Cotet CD, Palomba D, Barbui C, Cuijpers P. Efficacy of psychotherapies for borderline personality disorder: a systematic review and meta-analysis. JAMA Psychiatry 2017; 74(4): 319-28.

3 Storebø OJ, Stoffers-Winterling JM, Völlm BA, Kongerslev MT, Mattivi JT, Jørgensen MS, et al. Psychological therapies for people with borderline personality disorder. Cochrane Database Syst Rev 2020; 2020(5): CD012955.

4 coccaro EF, Harvey PD, Kupsaw-Lawrence E, Herbert JL, Bernstein D. Development of neuropharmacologically based behavioral assessments of impulsive aggressive behavior. J Neuropsychiatry Clin Neurosci 1991; 3(2): S44-51.

5 Pearce S, Scott L, Attwood G, Saunders K, Dean M, De Ridder R, et al. Democratic therapeutic community treatment for personality disorder: randomised controlled trial. Br J Psychiatry 2017; 210(2): 149-56.

6 Wetterborg D, Dehlbom P, Langstrom N, Andersson G, Fruzzetti AE, Enebrink P. Dialectical behavior therapy for men with borderline personality disorder and antisocial behavior: a clinical trial. J Pers Disord 2018; 34(1): 22-39.

7 Soler J, Pascual JC, Tiana T, Cebria A, Barrachina J, Campins MJ, et al. Dialectical behaviour therapy skills training compared to standard group therapy in borderline personality disorder: a 3-month randomised controlled clinical trial. Behav Res Ther 2009; 47(5): 353-8.

8 Neacsiu AD, Rizvi SL, Linehan MM. Dialectical behavior therapy skills use as a mediator and outcome of treatment for borderline personality disorder. Behav Res Ther 2010; 48(9): 832-9.

9 Bateman A, O'Connell J, Lorenzini N, Gardner T, Fonagy P. A randomised controlled trial of mentalization-based treatment versus structured clinical management for patients with comorbid borderline personality disorder and antisocial personality disorder. BMC Psychiatry 2016; 16: 304.

10 Mager W, Milich R, Harris MJ, Howard A. Intervention groups for adolescents with conduct problems: is aggregation harmful or helpful? J Abnorm Child Psychol 2005; 33(3): 349-62.

11 Gardner KJ, Archer J, Jackson S. Does maladaptive coping mediate the relationship between borderline personality traits and reactive and proactive aggression? Aggress Behav 2012; 38(5): 403-13.

12 Mancke F, Herpertz SC, Bertsch $\mathrm{K}$. Aggression in borderline personality disorder: a multidimensional model. Pers Disord Theory Res Treat 2015; 6(3): 278.

13 Bertsch K, Hillmann K, Herpertz SC. Behavioral and neurobiological correlates of disturbed emotion processing in borderline personality disorder. Psychopathology 2018; 51(2): 76-82.

14 Bertsch K, Roelofs K, Roch PJ, Ma B, Hensel S, Herpertz SC, et al. Neural correlates of emotional action control in anger-prone women with borderline personality disorder. J Psychiatry Neurosci 2018; 43(2): 170102.

15 Blair RJR. Traits of empathy and anger: implications for psychopathy and other disorders associated with aggression. Philos Trans R Soc Lond B Biol Sci 2018; 373(1744). Available from: https://doi.org/10.1098/rstb.2017.0155.

16 Mancke F, Herpertz SC, Kleindienst N, Bertsch K. Emotion dysregulation and trait anger sequentially mediate the association between borderline personality disorder and aggression. J Pers Disord 2017; 31(2): 256-72.

17 Sharp C, Kalpakci A. Mentalization in borderline personality disorder: from bench to bedside. Personal Disord 2015; 6(4): 347-55.

18 Herpertz SC, Jeung $\mathrm{H}$, Mancke F, Bertsch $\mathrm{K}$. Social dysfunctioning and brain in borderline personality disorder. Psychopathology 2014; 47(6): 417-24.

19 Berenson KR, Downey G, Rafaeli E, Coifman KG, Paquin NL. The rejection-rage contingency in borderline personality disorder. J Abnorm Psychol 2011; 120(3): $681-90$.

20 Berenson KR, Dochat C, Martin CG, Yang X, Rafaeli E, Downey G. Identification of mental states and interpersonal functioning in borderline personality disorder. Personal Disord 2018; 9(2): 172-81.

21 Schipper M, Petermann F. Relating empathy and emotion regulation: do deficits in empathy trigger emotion dysregulation? Soc Neurosci 2013; 8(1): 101-7.

22 Kazdin AE. Mediators and mechanisms of change in psychotherapy research. Annu Rev Clin Psychol 2007; 3: 1-27.

23 Linehan MM. Skills Training Manual for Treating Borderline Personality Disorder. Guilford Press, 1993.

24 Bateman AW, Fonagy P. Mentalization-based treatment of BPD. J Pers Disord 2004; 18(1): 36-51.

25 Macleod C, Grafton B. Regulation of emotion through modification of attention. In Handbook of Emotion Regulation (ed. Gross JJ): 508-28. Guilford Press, 2014.

26 Coccaro EF, Harvey PD, Kupsaw-Lawrence E, Herbert JL, Bernstein DP. Development of neuropharmacologically based behavioral assessments 
of impulsive aggressive behavior. J Neuropsychiatry Clin Neurosci 1991; 3(2): S44-51.

27 Coccaro EF, Lee RJ, Kavoussi RJ. A double-blind, randomized, placebocontrolled trial of fluoxetine in patients with intermittent explosive disorder. J Clin Psychiatry 2009; 70(5): 653-62.

28 Loranger AW. International Personality Disorder Examination: IPDE; DSM-IV and ICD-10; Interviews. PAR, 1999.

29 Zanarini MC, Vujanovic AA, Parachini EA, Boulanger JL, Frankenburg FR Hennen J. Zanarini Rating Scale for Borderline Personality Disorder (ZANBPD): a continuous measure of DSM-IV borderline psychopathology. J Pers Disord 2003; 17(3): 233-42.

30 Raven J. The Raven's progressive matrices: change and stability over culture and time. Cogn Psychol 2000; 41(1): 1-48.

31 Coccaro EF, Berman ME, Kavoussi RJ. Assessment of life history of aggression: development and psychometric characteristics. Psychiatry Res 1997; 73(3): 147-57.

32 Dandeneau SD, Baldwin MW. The inhibition of socially rejecting information among people with high versus low self-esteem: the role of attentional bias and the effects of bias reduction training. J Soc Clin Psychol 2004; 23(4): 584-603.

33 Izard C. Human Emotions. Plenum Press, 1977.

34 Neacsiu AD, Eberle JW, Kramer R, Wiesmann T, Linehan MM. Dialectical behavior therapy skills for transdiagnostic emotion dysregulation: a pilot randomized controlled trial. Behav Res Ther 2014; 59: 40-51.

35 McLaughlin SPB, Barkowski S, Burlingame GM, Strauss B, Rosendahl J. Group psychotherapy for borderline personality disorder: a meta-analysis of randomized-controlled trials. Psychotherapy 2019; 56(2): 260-73.

36 Cohen J. Statistical Power Analysis for the Behavioral Sciences (2nd edn). Lawrence Erlbaum Associates, 1988.

37 Cristea IA, Barbui C, Cuijpers P. Reviews and meta-analyses of psychotherapy efficacy for borderline personality disorder - reply. JAMA Psychiatry 2017; 74 (8): 854-5.

38 Barnicot K, Katsakou C, Marougka S, Priebe S. Treatment completion in psychotherapy for borderline personality disorder: a systematic review and meta-analysis. Acta Psychiatr Scand 2011; 123(5): 327-38.

39 Crawford MJ, Sanatinia R, Barrett B, Cunningham G, Dale O, Ganguli P, et al. The clinical effectiveness and cost-effectiveness of lamotrigine in borderline personality disorder: a randomized placebo-controlled trial. Am J Psychiatry 2018; 175(8): 756-64.
40 Stoffers J, Vollm BA, Rucker G, Timmer A, Huband N, Lieb K. Pharmacological interventions for borderline personality disorder. Cochrane Database Syst Rev 2010; 6: CD005653

41 Doering $\mathrm{S}$, Horz $\mathrm{S}$, Rentrop $\mathrm{M}$, Fischer-Kern $\mathrm{M}$, Schuster $\mathrm{P}$, Benecke $\mathrm{C}$, et al. Transference-focused psychotherapy $v$. treatment by community psychotherapists for borderline personality disorder: randomised controlled trial. $\mathrm{Br}$ Psychiatry 2010; 196(5): 389-95.

42 Robinson $P$, Hellier J, Barrett B, Barzdaitiene D, Bateman A, Bogaardt A, et al The NOURISHED randomised controlled trial comparing mentalisation-based treatment for eating disorders (MBT-ED) with specialist supportive clinical management (SSCM-ED) for patients with eating disorders and symptoms of borderline personality disorder. Trials 2016; 17(1): 549.

43 Hollander E, Tracy KA, Swann AC, Coccaro EF, McElroy SL, Wozniak P, et al. Divalproex in the treatment of impulsive aggression: efficacy in cluster B personality disorders. Neuropsychopharmacology 2003; 28(6): 1186-97.

44 Juarez-Trevino M, Esquivel AC, Isida LML, Delgado DAG, de la O Cavazos ME, Ocanas LG, et al. Clozapine in the treatment of aggression in conduct disorder in children and adolescents: a randomized, double-blind, controlled trial. Clin Psychopharmacol Neurosci 2019; 17(1): 43-53.

45 Williams RB, Williams VP. The prevention and treatment of hostility. In Handbook of Personality and Health (ed. Vollrath ME): 259-76. John Wiley Sons, 2006.

46 Chen C, Li C, Wang H, Ou JJ, Zhou JS, Wang XP. Cognitive behavioral therapy to reduce overt aggression behavior in Chinese young male violent offenders. Aggress Behav 2014; 40(4): 329-36.

47 Yen S, Johnson J, Costello E, Simpson EB. A 5-day dialectical behavior therapy partial hospital program for women with borderline personality disorder: predictors of outcome from a 3-month follow-up study. J Psychiatr Pract 2009; 15(3): 173-82.

48 Black DW, Allen J, St John D, Pfohl B, Mccormick B, Blum N. Predictors of response to Systems Training for Emotional Predictability and Problem Solving (STEPPS) for borderline personality disorder: an exploratory study. Acta Psychiatr Scand 2009; 120(1): 53-61.

49 National Collaborating Centre for Mental Health (UK). Borderline Personality Disorder: Treatment and Management. British Psychological Society, 2009.

50 Crawford MJ, Sanatinia R, Tan W. No effect of lamotrigine in subgroups of patients with borderline personality disorder: response to Smith. Am J Psychiatry 2018; 175(12): 1265-6. 